SPONDYLOEPIPHYSEAL OYSPLASIA CONGENITA: MORPHOLOGICAL AND BIOCHEMICAL STUDIES IN CUL TURED CHONDROCYTES.

Human chondrocytes dedifferentiate when they grow in monolayer cultures and produce type I collagen.

Re-differentiation is possible by culturing them in an agarose-gel. To characterize the modulated cells in more detail, ultrastructural and biochemical studies were performed in chondrocytes suspended in agarose for 2 weeks. It was shown that their ultrastructural characteristics were indistinguishable from those in tissue cells. Glycosaminoglcan (GAG) composition, electrophoretic mobility and hydrodynamic size of proteoglycans were identical in tissue and in cultured cells.

Chondrocytes from a patient with spondyloepiphyseal dysplasia congenita showed characteristic ultrastructural abnormalities both in biopsied cartilage and in cultured cells, most notably a dilated rough endoplasmic reticulum containing fine granular material. Proteoglycans produced by cultured cells contained abnormally high amounts of dermatan sulfate. The GAG chains were shorter than tose produced by normal cells. It remains to be established how these abnormalities are related to defects in collagen type II production that have been shown to exist in spondyloepiphyseal dysplasia congenita.

(Univ.-Kinderklinik, Langenbeckstraße 1, FRG - -6500 Mainz
MUSCLE PARALYSIS AS A LIFE-SAVING TREATMENT FOR LACTIC ACIDOSIS DUE TO A METABOLIC MUSCLE DISEASE.

68 J Faber, A Goren, $M$ Wllschanski, O Alpeleg, A Steinberg, B Ferber and $H$ Mandel. Departments of

Pediatries, Shaare Zedek, Bikur Holim and Rambam Medical Centers, Jerusalem and Haifa.

NR, a 14-year, old Moslem boy, had suffered from progressive tiredness and tachycardia for 7-years. Investigations ruled out primary heart disease or endocrinopathy. High cardiac output at rest and lactic acidosis after minimal exertion were found. Muscle biopsy showed an undefined myopathy and glycogen accumulation. His last admission was because of severe lactic acidosis unresponsive to large doses of bicarbonate. Dur suspicion of a defect in the mitochondrial respiratory chain of the muscle was supported by NR's urinary profile of organic acids. Pharmacological doses of vitamins $K_{1} B_{2}, B_{12}$, $B_{6}$ and niacin were ineffective, and severe congestive heart failure developed. Therefore, total muscle paralysis was pharmacologically induced while NR was put on artificial respiration. Within six hours acidosis began to improve and $4 \theta$ hours later muscle paralysis was discontinued. Thereafter NR made a slow recovery to his original condition.

In summary: This case-report demonstrates that minimizing energy needs by muscle paralysis may be life-saving in cases of lactic acidosis due to a mitochondrial myopatny.
Clinical usefulness of the genetis studies in galactosemic families.

66

\section{B. Radomyska}

National Research Institute of Mother and Child, Warsaw, Poland

2 groups of families were examined: I-31 in which a case of galactosemia has occured. II-21 families of children with very low uridyl transferase detected in neonatal screening test.

On the basis of genetic studies /analysis of pedigrees, uridyl transferase activity measurement/, the heterozygotes entered the genetic counselling. The ear Iy prophylaxis/galactose-freo diet/ had been applied in every pregnancy when both parents had been known as heterozygotes and for every newborn from the very begining. Newborns were diagnosed in the first day of life.

The genetic studies in galactosemic families allowed: - to exclude mortality from galactosemia in these families

- to eliminate serious consequences of the disease caused by late diagnosis

- to achieve normal development of heterozygotes born in families which underwent the genetic counselling.

\section{7}

THE USE OF CDNA PROBES FOR DETECTION OF DELETIONS IN DNA OF DUCHENNE MUSCULAR DYSTROPHY BOYS.

E. Popowska, J. Popowski, M. Krajewska-Walasek,

E. Pronicka

Child Health Centre, Warsaw, Poland

Duchenne muscular dystrophy (DMD) is X-linked recessive neuromuscular disease mapped in band Xp21. Since 1983 linkage of the disease to restriction fragment length polymorphism (RLFP) has been applied for genetic counseling. Recently DMD cDNA has been cloned so the possibility of direct detection of DMD gene mutation appears. The purpose of our study was to examine usefulness of cDNA probes for detection of deletions in DNA of polish patients.

Eleven DNA samples of DMD boys from 10 families were digested with enzyme Hind III and hybridized to labelled cDNA probes: $1-2 \mathrm{a}, 5 \mathrm{~b}-7$ and 8 , supplied by ATCC.

In 5 out of total 11 DNA samples partial DMD gene deletions were found: in 2 patients it was located in the middle and in 3 patients on the proximal portion of the gene.

Our study suggests the testing all affected boys with cDNA probes for deletions is more cost-effective and faster than RLFP analysis. Besides in $40 \%$ of cases it provides an accurate genetic marker for subsequent prenatal diagnosis in the families.
INFANTTEE CATARACT WITH TRANSIENT INCREASE OF SERUM GAIACTITOL LEVELS.

69 H. Gerding, K. Ullrich, Y. Shin ${ }^{+}, \mathrm{C}$. Jacobs ${ }^{++}$ Depts. of Ophthalmology and Pediatrics, University of Münster, München and Amsterdam

Two patients (P1: 1 month, P2: 2 years old) with polar cataract of one eye were observed. P2 underwent cataract surgery to prevent amblyopia. The neurological development corresponced to age.

The enzymatic activities of galactokinase, transferase, epimerase and sorbitdehydrogenase were found to be normal in erythrocytes of $P 1$ and P2, such as cultivated fibroblasts of P1. In both patients normal concentrations of galactose (serum) and galactose1-phosphate (exythrocytes) were found. During a galactose loading test ( $1 \mathrm{~g} / \mathrm{kg}$ b. w.) the increase of galactose and galactose-1phosphate concentrations was within the normal range. Serum galactitol levels were increased $(P 1=2,65, P 2=1,08 \mu \mathrm{mol}$; normal: $0,09-0,52 \mu \mathrm{mol})$ whereas serum sorbitol levels were normal $(<3,08 \mu \mathrm{mol})$. One and five months after introduction of a galactose-free diet normal serum galactitol levels were found, remaining on the same level now up to one year after termination of the diet by the families $(0,29$ and $0,3 \mu \mathrm{mol}$, respectively). Accumulation of galactitol in the lens possibly causes cataract. The mechanism of the serum galactitol elevation of our patients remains unclear. A transient increase of aldosereductase activity may be discussed. \section{Bocian E., Maciejko D., Mazurczak T, Bal J., Bulawa E. \\ 70 Institute of Mother and Child, Warsaw, Poland}

Data on fragile $X$ ( $($ 227.3) site expression in 5 families suspected for $X$-linked mental retardation are presented. Chromosomal analysis was performed in 30 persons, 16 males and 14 females. Fragile site expression was induced in thymidylate stress conditions achieved by the culture of lymphocytes in folic acid defficient medium 199 or by the introduction of methotrexate to standard Eagle's (MEM) medium. Of the 21 fra $(\mathrm{X})$ positive persons 13 were males and 8 were females. Expression of the fragile site could not be demonstrated in 3 out of 8 female obligate carrier. Mean frequency of fra (X) site expression was approximately 2 -fold higher, when methotrexate was present in the walture medium ( $32.7 \%$ for males and $8.8 \%$ for females). No relation could culture medium ( $32.7 \%$ for males and $8.8 \%$ for females). No relation co
be found between the age of the fragile $\mathrm{X}$ carrier and expression of the fragility.

RLFP analysis was performed in 4 families. In one informative family problematic cytogenetic diagnosis of fra-X carriership was elucidated. 